\title{
Analysis of c-KIT expression and KIT gene mutation in human mucosal melanomas
}

\author{
I Satzger ${ }^{*,}$, T Schaefer', U Kuettler', V Broecker², B Voelker', H Ostertag ${ }^{3}$, A Kapp' and R Gutzmer' \\ 'Department of Dermatology and Allergology, Skin Cancer Center Hannover, Hannover Medical School, Hannover, Germany; ${ }^{2}$ Department of Pathology, \\ Hannover Medical School, Hannover, Germany; ${ }^{3}$ Department of Pathology, Klinikum Region Hannover, Hannover, Germany
}

Recent data suggested an increased frequency of KIT aberrations in mucosal melanomas, whereas c-KIT in most types of cutaneous melanomas does not appear to be of pathogenetic importance. However, studies investigating the status of the KIT gene in larger, well-characterised groups of patients with mucosal melanomas are lacking. We analysed 44 archival specimens of 39 wellcharacterised patients with mucosal melanomas of different locations. C-KIT protein expression was determined by immunhistochemistry, KIT gene mutations were analysed by PCR amplification and DNA sequencing of exons 9, II, I3, I7 and 18. c-KIT protein expression could be shown in 40 out of 44 (91\%) tumours in at least 10\% of tumour cells. DNA sequence analysis of the KIT was successfully performed in 37 patients. In 6 out of 37 patients (I6\%) KIT mutations were found, five in exon II and one in exon 18. The presence of mutations in exon II correlated with a significant stronger immunohistochemical expression of c-KIT protein $(P=0.015)$. Among the six patients with mutations, in two patients the primary tumour was located in the head/neck region, in three patients in the genitourinary tract and in one patient in the anal/rectal area. In conclusion, KIT mutations can be found in a subset of patients with mucosal melanomas irrespective of the location of the primary tumour. Our data encourage therapeutic attempts with tyrosine kinase inhibitors blocking c-KIT in these patients.

British Journal of Cancer (2008) 99, 2065-2069. doi:I0.1038/sj.bjc.660479I www.bjcancer.com

Published online 18 November 2008

(c) 2008 Cancer Research UK

Keywords: melanoma; mucosal; c-KIT; KIT; BRAF

Primary mucosal melanomas represent a rare subtype of melanomas that arise from melanocytic cells residing in mucous membranes in various anatomical regions. They account for approximately $1.2 \%$ of melanomas (Chang et al, 1998) and have mainly been described in the head and neck region, the genitourinary tract and the gastrointestinal tract.

Obviously, sun exposure does not play a role in mucosal melanoma, whereas it is a risk factor in cutaneous melanoma. A recent study described genetic differences between mucosal melanomas and cutaneous melanomas. In this study, four types of melanomas were differentiated, that is, mucosal melanomas, acral melanomas, melanomas on skin with chronic sun damage and melanomas on skin without chronic sun damage. Melanomas arising from skin without chronic sun damage, representing the major group of cutaneous melanomas, were shown to frequently harbour BRAF mutations, in particular the BRAF V600E mutation. The other melanoma types, including mucosal melanoma, had a high frequency of mutations of the KIT gene (Curtin et al, 2005, 2006). This finding is in particular intriguing as it may represent a rationale for a targeted therapy with specific tyrosine kinase inhibitors such as c-KIT blockers in mucosal melanomas. To

*Correspondence: Dr I Satzger, Department of Dermatology and Allergology, Skin Cancer Center Hannover, Hannover Medical School, Ricklinger Str. 5, D-30449 Hannover, Germany;

E-mail: satzger.imke@mh-hannover.de

Received 25 July 2008; revised 27 October 2008; accepted 27 October 2008; published online 18 November 2008 further elucidate the role of c-KIT and $B R A F$ in mucosal melanoma, we analysed these two targets in 39 patients with mucosal melanomas treated in our Department.

\section{MATERIALS AND METHODS}

\section{Patients}

Thirty nine patients with mucosal melanomas who were treated in our Department (Skin Cancer Center Hannover) from 1996 to 2007 were retrospectively analysed. A total of 44 archival formalin-fixed and paraffin-embedded tissue samples (35 primary melanomas, 4 lymph node metastases, 2 skin metastases and 3 local recurrences) were accessible for analysis in this study.

\section{Immunohistopathologic evaluation of c-KIT expression}

Highly sensitive immunohistochemistry for c-KIT with a murine monoclonal antibody (clone p145, dilution 1:100, DakoCytomation, Hamburg, Germany) was performed as described earlier (Satzger et al, 2008) and immunohistochemical stainings were evaluated semiquantitatively. The numbers of positively labelled melanoma cells were scored as follows: 0 for less than $10 \%$ positive cells, $1(+)$ for $10-25 \%$ positive cells, $2(++)$ for $26-50 \%$ positive cells, $3(+++)$ for $51-75 \%$ and $4(++++)$ for $76-$ $100 \%$ positive cells. We did not differentiate between cytoplasmatic and membranous staining as we did not observe isolated surface staining and earlier studies showed cytoplasmatic 
staining along with membranous staining in melanoma cells positive for c-KIT (Pereira et al, 2005; Giehl et al, 2007; Rivera et al, 2008).

\section{Mutational analysis of KIT}

Tumour cells were isolated from paraffin-embedded tissue (either primary tumour, lymph node metastases, skin metastases or local recurrences), if necessary by micrographic dissection using the PALM Laser-MicroBeam System (PALM Wolfratshausen, Germany). DNA extraction was performed with the DNA extraction kit from Qiagen (Hilden, Germany) following the instructions of the manufacturer. Exons 9, 11, 13, 17, 18 of KIT were amplified by LightCycler PCR using specific primers as described in the literature (Tarn et al, 2005; Curtin et al, 2006). Polymerase chain reaction products were DNA sequenced using an ABI Prism 3700 DNA Analyzer (SeqLab, Göttingen, Germany).

\section{Mutational analysis of BRAF}

To detect the BRAF V600E mutation a LightCycler fluorescence resonance energy transfer (FRET) assay with two fluorescent hybridisation probes was performed as described earlier (Hay et al, 2007). Real-time PCR was performed by using LightCycler FastStart DNA Master HybProbe (Roche Diagnostics $\mathrm{GmbH}$, Mannheim, Germany). Post amplification fluorescent melting curve analysis was performed by gradual heating of the samples at a rate of $0.2^{\circ} \mathrm{C}$ per second from 45 to $95^{\circ} \mathrm{C}$. Fluorescent melting peaks were determined by plotting of the negative derivative of fluorescence with respect to temperature.

All PCR products that showed deviation from the Wt (wild-type) genomic DNA melting peak as well as from the positive control samples were confirmed by direct sequencing of exon 15 of the $B R A F$ gene (SequiServe, Vaterstetten, Germany).

\section{Statistical analyses}

The software SPSS 13.0 was used for statistical analyses. KaplanMeier tests and unpaired $t$-tests were performed.

\section{RESULTS}

c-KIT expression was determined in 44 tissue samples, 35 primary mucosal melanomas, 4 lymph node metastases, 2 skin metastases and 3 local recurrences (Table 1). In all, 31 out of $35(89 \%)$ primaries, 4 out of $4(100 \%)$ lymph node metastases, 2 out of 2 $(100 \%)$ skin metastases and 3 out of $3(100 \%)$ local recurrences were positive for c-KIT (Figure 1A). c-KIT protein expression could be detected in 18 out of $18(100 \%)$ mucosal melanomas located in the head/neck area, 7 out of $8(86 \%)$ in the anal/rectal tract, 8 out of $11(73 \%)$ in the genitourinary tract and 2 out of 2 $(100 \%)$ in other locations. Score $1+$ was determined in 8 out of 44 samples $(18 \%)$, score $2+$ in 7 out of $44(16 \%)$ samples, score $3+$ in 9 out of $44(20 \%)$ samples and score $4+$ in 16 out of 44 samples $(36 \%)$. The degree of the c-KIT expression did not correlate with disease-free survival $(P=0.44)$ and overall survival $(P=0.82)$ by Kaplan-Meier analysis during a follow-up of 31.1 months (mean) and 21.1 months (median, minimum 2.8 months, maximum 151.6 months). During that follow-up period, 20 patients suffered from melanoma recurrence and 14 patients died due to melanoma metastases.

Alterations in the KIT gene were observed in 6 out of $37(16 \%)$ patients, five in exon 11 and one in exon 18 (Figure 1B). Two patients suffered from mucosal melanomas of the head/neck region, three patients from mucosal melanomas located in the genitourinary tract and one patient from mucosal melanoma located in the anal/rectal tract. In one patient (case 27) the KIT mutation could be detected both in lymph node metastases and in skin metastases. Among the five tumours with KIT gene mutation of exon 11 , four $(80 \%)$ tumours showed strong $(++++)$ and one showed $(20 \%)$ high $(+++)$ c-KIT protein expression (Figure 1A). In contrast, tumours without mutation in exon 11 had significantly lower c-KIT expression ( 3 out of 32 negative, 7 out of $32(+), 7$ out of $32(++), 8$ out of $32(+++), 7$ out of 32 $(++++), P=0.015)$.

In 3 out of $27(11 \%)$ mucosal melanomas (located in head/neck area, pleura and conjunctiva, respectively) the $B R A F \mathrm{~V} 600 \mathrm{E}$ mutation could be detected (Table 1, Figure 1C).

\section{DISCUSSION}

A recent report showed a possible role of c-KIT in subsets of melanoma, in particular, mucosal melanomas ( $21 \%$ KIT mutations, $61 \%$ c-KIT overexpression), acral cutaneous melanomas (11\% KIT mutations, $75 \%$ c-KIT overexpression) and cutaneous melanomas on skin with chronic sun damage (17\% KIT mutations, $100 \%$ c-KIT overexpression) (Curtin et al, 2006). This suggests that c-KIT might be of pathogenetic relevance and therefore a therapeutic target in these subtypes of melanoma. In contrast, KIT mutations are rarely found in the major subtype of cutaneous melanoma originating from skin without chronic sun damage (Curtin et al, 2006) and unselected cutaneous melanomas (2 out of 100 in Willmore-Payne et al (2005); 1 out of 39 in Went et al (2004)). Moreover, therapeutic phase II studies with the c-KIT blocker imatinib in unselected melanoma patients without known KIT mutation status were disappointing (Ugurel et al, 2005; Wyman et al, 2006; Becker et al, 2007).

The aim of this study was to further elucidate c-KIT alterations in our well-characterised group of patients with mucosal melanomas that might support the role of c-KIT as a new therapeutic target in this subgroup of melanoma. The successful genetic analysis of KIT in 37 patients revealed mutations in 6 patients (16\%). We could show KIT mutations in 2 out of 12 mucosal melanomas from head/neck, 3 out of 11 from the genitourinary tract and 1 out of 8 from the anal/rectal tract. This is consistent with the findings of Antonescu et al and Rivera et al who detected mutations of the KIT gene in 3 out of 20 (15\%) and 4 out of $18(22 \%)$ patients with mucosal melanomas of the anal region and oral cavity, respectively (Antonescu et al, 2007; Rivera et al, 2008). Thus, KIT mutations occur in up to $20 \%$ of mucosal melanomas irrespective of the location of the primary tumour.

The majority of KIT mutations in mucosal melanomas (11 out of 16 tumours in Curtin et al (2006), 3 out of 3 in Antonescu et al (2007), 4 out of 4 in Rivera et al (2008) and 5 out of 6 in our study) were detected in the juxtamembrane region of KIT encoded by exons 11 and 13, presumably resulting in the activation of c-KIT.

The L576P und W557R mutations of our patients have already been described both in mucosal melanomas and gastrointestinal stromal tumours (GIST) (Antonescu et al, 2004, 2007; Rivera et al, 2008). Deletions covering the 579 position (such as in our patient 24) have also been frequently described in GIST (Tarn et al, 2005). The $\mathrm{K} 550 \mathrm{~N}$ mutation found in our patient 2 has, to our knowledge, not been described yet. However, other alterations in the proximal part of exon 11 at codons 550-562 have been reported frequently in GIST (Lasota et al, 1999; Longley et al, 2001).

Thus, the minor subgroup of patients with mucosal melanomas and activating KIT mutations might be susceptible to therapeutic c-KIT blockade. This is supported by findings in GIST, which show KIT mutations in $75-80 \%$, and respond significantly better to a therapy with the c-KIT inhibitor imatinib than tumours without KIT mutations (Heinrich et al, 2003). Therefore, therapeutic c-KIT blockade could be considered for the treatment of patients with 
Table I Characterisation of patients, expression of c-KIT by immunohistochemistry (IHC), mutational status of KIT and BRAF in mucosal melanoma, clinical follow-up

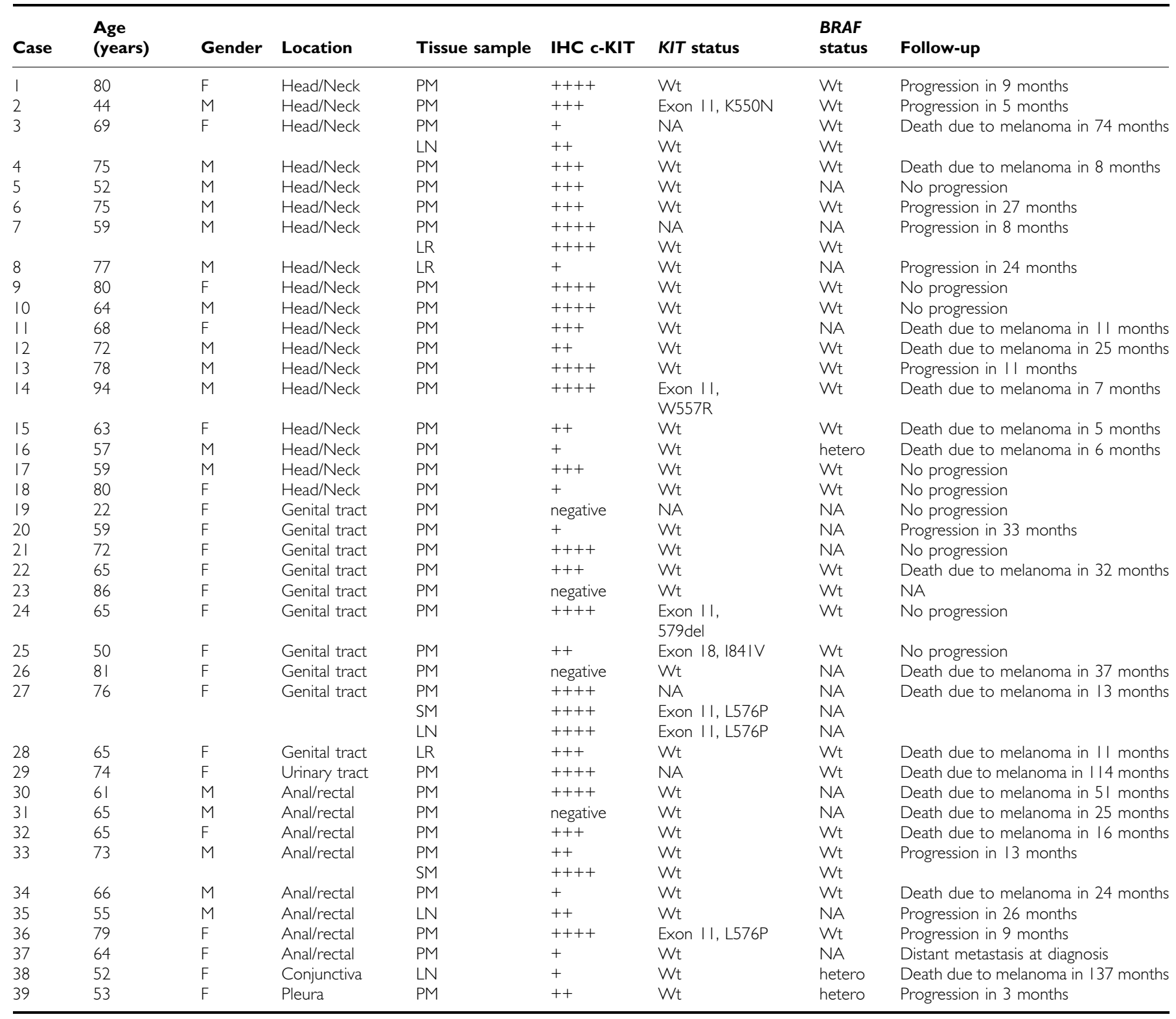

hetero = heterozygous, $L N=$ lymph node metastasis; $L R=$ local recurrence, $N A=$ not available, $P M=$ primary melanoma, $S M=$ skin metastasis, $W t=$ wild-type. $I H C$ assessment: negative for less than $10 \%$ positive cells, + for $10-25 \%$ positive cells, ++ for $25-50 \%$ positive cells, +++ for $50-75 \%$ and ++++ for 75 to $100 \%$ positive cells.

mucosal melanomas and an activating KIT mutation. This is supported by two case reports published very recently of single patients suffering from metastasising anal melanoma that harboured a KIT mutation in exon 11 and exon 13, respectively. These patients were successfully treated with the c-KIT blocker imatinib (Hodi et al, 2008; Lutzky et al, 2008).

c-KIT protein expression could be observed in $91 \%$ of our primary mucosal melanomas, which is similar to the rates reported in other series of primary mucosal melanomas of the anal/rectal tract (12 out of 16 in Chute et al, 2006) and oral cavity (16 out of 18 in Rivera et al, 2008), respectively. However, Antonescu et al (2007) reported lower number (6 out of 26 (23\%)) of c-KIT positivity in mucosal melanomas of the anal/rectal tract. In addition, cutaneous melanomas have been reported to express c-KIT by immunohistochemistry in $22.8 \%$ (Potti et al, 2003) up to $84 \%$ (Giehl et al, 2007) of the cases. The high range between these studies is being explained with the different qualities of immunohistochemistry. Thus, c-KIT protein expression appears to be in a similar range in mucosal melanomas and cutaneous melanomas. In line with this, Giehl et al could find no differences of the c-KIT expression in melanomas with sun exposure compared with melanomas without sun exposure (Giehl et al, 2007). In contrast to metastatic melanoma from primary cutaneous melanoma, where the c-KIT expression is decreased (Montone et al, 1997; Shen et al, 2003), we could observe c-KIT expression in 9 out of 9 metastases of primary mucosal melanoma. Similarly, Antonescu et al detected c-KIT reactivity in 6 out of 6 metastases from their anal mucosal melanomas (Antonescu et al, 2007). Furthermore, in agreement with earlier studies we could not show a prognostic relevance of the c-KIT protein expression in mucosal melanomas (Chute et al, 2006).

We could observe a significant higher immunohistochemical expression of c-KIT in mucosal melanomas that harbour a potentially activating KIT mutation as compared with tumours 

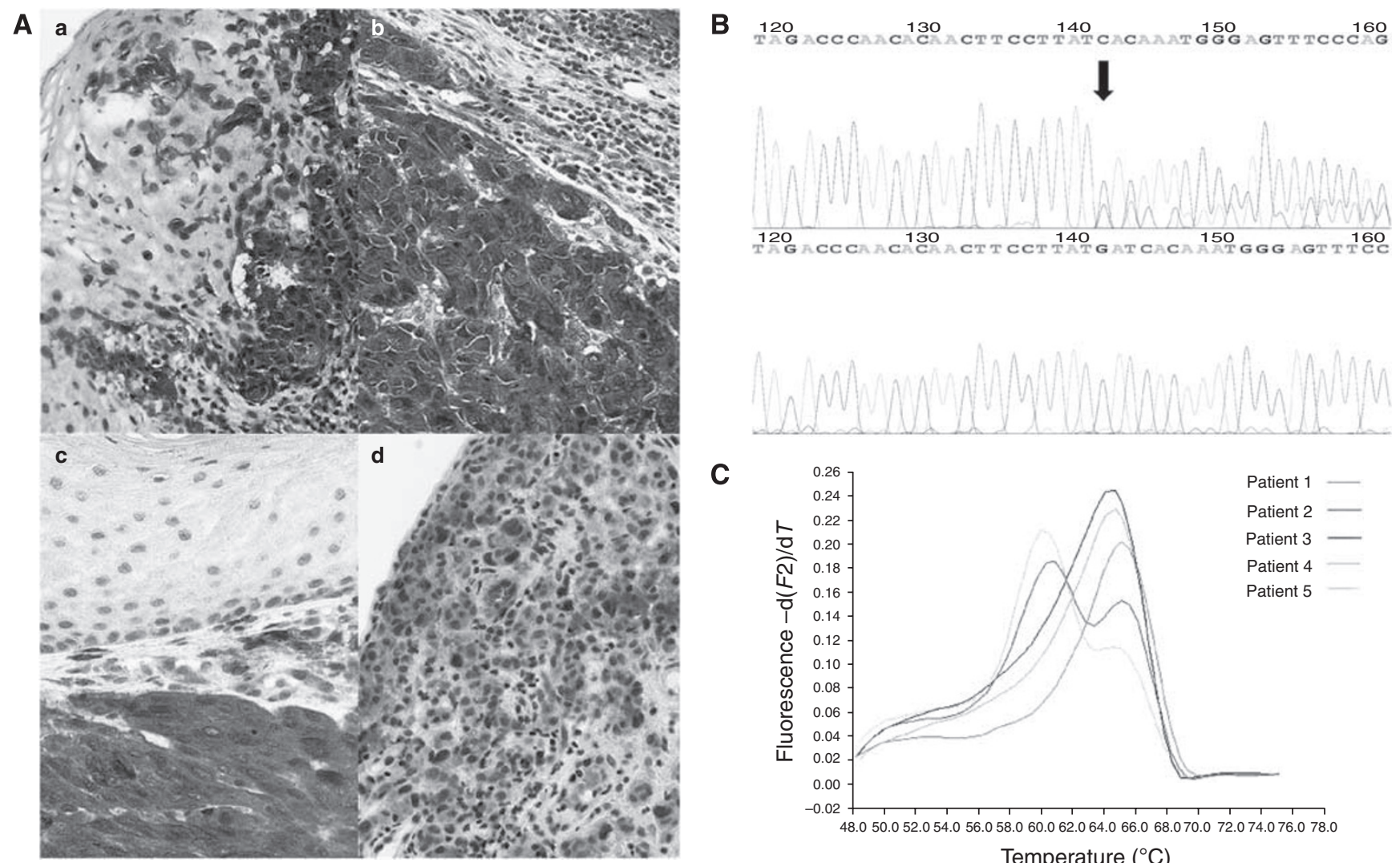

Figure I (A) Immunhistochemical staining of c-KIT in primary mucosal melanoma showing strong positive membranous and cytoplasmatic labelling $(++++)$ of primary melanoma $(\mathbf{a})$, lymph node metastases $(\mathbf{b})$ and skin metastases $(\mathbf{c})$ of patient 27 (magnification $\times 100)$ and, weak and inhomogeneous labelling $(+)$ of primary melanoma $(\mathbf{d})$ of patient 18 (magnification $\times 100)$. (B) KIT genotyping shows a deletion 579 in exon I I (patient 24). The upper curve depicts the KIT exon II forward sequence and the lower curve depicts the wild-type sequence. (C) Melting curve analysis of five patients with mucosal melanomas. Melting curve peaks at $64.7^{\circ} \mathrm{C}$ from BRAF wild type (Patient nos. I, 3 and 4 , corresponding to patients I, 3 and 4 in Table I) and melting curve peak at $60.6^{\circ} \mathrm{C}$ from BRAF V600E heterozygous mutation (cases 2 and 5 , corresponding to cases 16 and 38 in Table I).

without KIT mutation. A similar correlation has also been found for anal melanomas (Antonescu et al, 2007) and melanomas of the oral cavity (Rivera et al, 2008).

Thus, immunohistochemistry might be a useful tool to screen for patients that are subjected to KIT mutation analysis. However, immunohistochemistry might not be sufficient to detect tumours with mutations susceptible for c-KIT blockade, as overexpression of c-KIT can also occur in tumours without mutation. This is illustrated by the report of three patients with metastatic melanoma and strong immunohistochemical c-KIT expression who did not respond to a therapy with the c-KIT blocker imatinib (Alexis et al, 2005).

$B R A F$ mutations were rarely identified in 3 out of $27(11 \%)$ patients in our series, similarly to other studies with patients suffering from mucosal melanomas who reported 1 out of 17 (Cohen et al, 2004) and 0 out of 13 (Edwards et al, 2004), respectively, whereas the majority of cutaneous melanomas on skin without chronic sun-induced damage harbour BRAF V600E mutations (Curtin et al, 2005). It is interesting to note that one of our patients with a $B R A F$ mutation suffered from melanoma located in an UV-exposed area of the conjunctiva. In line with this, Gear et al (2004) reported BRAF mutations in 5 out of 22 patients with conjunctival melanomas.

In conclusion, our study supports the finding that KIT mutations presumably activating the tyrosine kinase activity of c-KIT can be found in a subgroup of patients with mucosal melanomas irrespective of the origin of the primary tumour. This encourages clinical studies with c-KIT blockers in patients with mucosal melanomas and appropriate KIT mutations. Immunohistochemistry for c-KIT expression might be a useful tool to screen for patients who are subjected to mutational analysis but cannot replace genetic analysis.

\section{ACKNOWLEDGEMENTS}

This study was supported by Hannelore-Munke research grant of Hannover Medical School (Hannover, Germany).

\section{REFERENCES}

Alexis JB, Martinez AE, Lutzky J (2005) An immunohistochemical evaluation of c-kit (CD-117) expression in malignant melanoma, and results of imatinib mesylate (Gleevec) therapy in three patients. Melanoma Res 15: 283 - 285

Antonescu CR, Busam KJ, Francone TD, Wong GC, Guo T, Agaram NP, Besmer P, Jungbluth A, Gimbel M, Chen CT, Veach D, Clarkson BD, Paty
PB, Weiser MR (2007) L576P KIT mutation in anal melanomas correlates with KIT protein expression and is sensitive to specific kinase inhibition. Int J Cancer 121: $257-264$

Antonescu CR, Viale A, Sarran L, Tschernyavsky SJ, Gonen M, Segal NH, Maki RG, Socci ND, DeMatteo RP, Besmer P (2004) Gene expression in 
gastrointestinal stromal tumors is distinguished by KIT genotype and anatomic site. Clin Cancer Res 10: 3282-3290

Becker JC, Brocker EB, Schadendorf D, Ugurel S (2007) Imatinib in melanoma: a selective treatment option based on KIT mutation status? $J$ Clin Oncol 25: e9

Chang AE, Karnell LH, Menck HR (1998) The National Cancer Data Base report on cutaneous and noncutaneous melanoma: a summary of 84,836 cases from the past decade. The American College of Surgeons Commission on Cancer and the American Cancer Society. Cancer 83: 1664-1678

Chute DJ, Cousar JB, Mills SE (2006) Anorectal malignant melanoma: morphologic and immunohistochemical features. Am J Clin Pathol 126: $93-100$

Cohen Y, Rosenbaum E, Begum S, Goldenberg D, Esche C, Lavie O, Sidransky D, Westra WH (2004) Exon 15 BRAF mutations are uncommon in melanomas arising in nonsun-exposed sites. Clin Cancer Res 10: $3444-3447$

Curtin JA, Busam K, Pinkel D, Bastian BC (2006) Somatic activation of KIT in distinct subtypes of melanoma. J Clin Oncol 24: 4340-4346

Curtin JA, Fridlyand J, Kageshita T, Patel HN, Busam KJ, Kutzner H, Cho KH, Aiba S, Brocker EB, LeBoit PE, Pinkel D, Bastian BC (2005) Distinct sets of genetic alterations in melanoma. $N$ Engl J Med 353: 2135-2147

Edwards RH, Ward MR, Wu H, Medina CA, Brose MS, Volpe P, Nussen-Lee S, Haupt HM, Martin AM, Herlyn M, Lessin SR, Weber BL (2004) Absence of BRAF mutations in UV-protected mucosal melanomas. J Med Genet 41: $270-272$

Gear H, Williams H, Kemp EG, Roberts F (2004) BRAF mutations in conjunctival melanoma. Invest Ophthalmol Vis Sci 45: 2484-2488

Giehl KA, Nagele U, Volkenandt M, Berking C (2007) Protein expression of melanocyte growth factors (bFGF, SCF) and their receptors (FGFR-1, c-kit) in nevi and melanoma. J Cutan Pathol 34: 7-14

Hay R, MacRae E, Barber D, Khalil M, Demetrick DJ (2007) BRAF mutations in melanocytic lesions and papillary thyroid carcinoma samples identified using melting curve analysis of polymerase chain reaction products. Arch Pathol Lab Med 131: 1361-1367

Heinrich MC, Corless CL, Demetri GD, Blanke CD, von Mehren M, Joensuu $\mathrm{H}$, McGreevey LS, Chen CJ, Van den Abbeele AD, Druker BJ, Kiese B, Eisenberg B, Roberts PJ, Singer S, Fletcher CD, Silberman S, Dimitrijevic $S$, Fletcher JA (2003) Kinase mutations and imatinib response in patients with metastatic gastrointestinal stromal tumor. J Clin Oncol 21: $4342-4349$

Hodi FS, Friedlander P, Corless CL, Heinrich MC, Mac RS, Kruse A, Jagannathan J, Van den Abbeele AD, Velazquez EF, Demetri GD, Fisher DE (2008) Major response to imatinib mesylate in KIT-mutated melanoma. J Clin Oncol 26: 2046-2051

Lasota J, Jasinski M, Sarlomo-Rikala M, Miettinen M (1999) Mutations in exon 11 of c-Kit occur preferentially in malignant versus benign gastrointestinal stromal tumors and do not occur in leiomyomas or leiomyosarcomas. Am J Pathol 154: 53-60

Longley BJ, Reguera MJ, Ma Y (2001) Classes of c-KIT activating mutations: proposed mechanisms of action and implications for disease classification and therapy. Leuk Res 25: 571-576

Lutzky J, Bauer J, Bastian B (2008) Dose-dependent, complete response to imatinib of a metastatic mucosal melanoma with a K642E KIT mutation. Pigment Cell Melanoma Res 24: 491-493

Montone KT, Van Belle P, Elenitsas R, Elder DE (1997) Proto-oncogene c-kit expression in malignant melanoma: protein loss with tumor progression. Mod Pathol 10: 939-944

Pereira PR, Odashiro AN, Marshall JC, Correa ZM, Belfort Jr R, Burnier Jr MN (2005) The role of c-kit and imatinib mesylate in uveal melanoma. J Carcinog 4: 19

Potti A, Hille R, Koch M (2003) Immunohistochemical determination of HER-2/neu in malignant melanoma. Anticancer Res 23: 4067-4069

Rivera RS, Nagatsuka H, Gunduz M, Cengiz B, Gunduz E, Siar CH, Tsujigiwa H, Tamamura R, Han KN, Nagai N (2008) C-kit protein expression correlated with activating mutations in KIT gene in oral mucosal melanoma. Virchows Arch 452: $27-32$

Satzger I, Meier A, Gutzmer R (2008) The Prognostic Significance of Isolated HMB-45 or Melan-A-Positive Cells in Melanoma Sentinel Lymph Node. Am J Surg Pathol

Shen SS, Zhang PS, Eton O, Prieto VG (2003) Analysis of protein tyrosine kinase expression in melanocytic lesions by tissue array. J Cutan Pathol 30: $539-547$

Tarn C, Merkel E, Canutescu AA, Shen W, Skorobogatko Y, Heslin MJ, Eisenberg B, Birbe R, Patchefsky A, Dunbrack R, Arnoletti JP, von Mehren M, Godwin AK (2005) Analysis of KIT mutations in sporadic and familial gastrointestinal stromal tumors: therapeutic implications through protein modeling. Clin Cancer Res 11: 3668-3677

Ugurel S, Hildenbrand R, Zimpfer A, La Rosee P, Paschka P, Sucker A, Keikavoussi P, Becker JC, Rittgen W, Hochhaus A, Schadendorf D (2005) Lack of clinical efficacy of imatinib in metastatic melanoma. Br J Cancer 92: $1398-1405$

Went PT, Dirnhofer S, Bundi M, Mirlacher M, Schraml P, Mangialaio S, Dimitrijevic S, Kononen J, Lugli A, Simon R, Sauter G (2004) Prevalence of KIT expression in human tumors. J Clin Oncol 22: 4514-4522

Willmore-Payne C, Holden JA, Tripp S, Layfield LJ (2005) Human malignant melanoma: detection of BRAF- and c-kit-activating mutations by high-resolution amplicon melting analysis. Hum Pathol 36: $486-493$

Wyman K, Atkins MB, Prieto V, Eton O, McDermott DF, Hubbard F, Byrnes C, Sanders K, Sosman JA (2006) Multicenter Phase II trial of highdose imatinib mesylate in metastatic melanoma: significant toxicity with no clinical efficacy. Cancer 106: 2005-2011 